Research report

\title{
Religious attendance and social adjustment as protective against depression: A 10-year prospective study
}

\author{
Yakov A. Barton a , Lisa Miller ${ }^{\mathrm{a}, \mathrm{b}}$, Priya Wickramaratne ${ }^{\mathrm{b}, \mathrm{c}, \mathrm{d}}$, Marc J. Gameroff ${ }^{\mathrm{b}, \mathrm{c}}$, \\ Myrna M. Weissman ${ }^{\mathrm{b}, \mathrm{c}, \mathrm{d}, *}$ \\ ${ }^{a}$ Columbia University, Teachers College, USA \\ ${ }^{\mathrm{b}}$ Columbia University, College of Physicians E Surgeons, USA \\ ${ }^{\mathrm{c}}$ New York State Psychiatric Institute, USA \\ d Columbia University, Mailman School of Public Health, USA
}

\section{A R T I C L E I N F O}

\section{Article history:}

Received 21 August 2012

Accepted 22 August 2012

Available online 7 September 2012

\section{Keywords}

Major depression

Religiosity

Religious attendance

Social adjustment

\begin{abstract}
A B S T R A C T
Background: Previous research has identified elevated social adjustment and frequent religious attendance as protective against depression. The present study aims to examine the association of frequency of religious services attendance with subsequent depression, while accounting for the effects of social adjustment.

Method: Participants were 173 adult offspring of depressed and nondepressed parents, followed longitudinally over 25 years. Diagnosis was assessed with the Schedule for Affective Disorders and Schizophrenia-Lifetime Version. The Social Adjustment Scale-Self Report (SAS-SR) was used to assess social adjustment and frequency of religious services attendance was self-reported. In a logistic regression analysis, major depression at 20 years was used as the outcome measure and the frequency of religious services attendance and social adjustment variables at 10 years as predictors.

Results: Frequent religious services attendance was found to protect against subsequent depression at a trend level. High functioning social adjustment was found to protect against subsequent depression, especially within the immediate and extended family. Adults without a depressed parent who reported attending religious services atleast once a month had a lower likelihood of subsequent depression. Among adults with a depressed parent, those with high functioning social adjustment had a lower likelihood of subsequent depression.

Limitations: Measurement of social adjustment was non-specific to religious services.

Conclusions: Frequent religious attendance may protect against major depression, independent from the effects of social adjustment. This protective quality may be attenuated in adults with a depressed parent. High functioning social adjustment may be protective only among offspring of depressed parents.
\end{abstract}

(c) 2013 Elsevier B.V. All rights reserved.

\section{Introduction}

Research examining protective factors against major depression has identified frequency of religious services attendance (Hayward et al., 2012a; Koenig, 2007; Maselko et al., 2009; Norton et al., 2008; Sternthal et al., 2010) and social support (Bruce and Hoff, 1994; De Leeuw et al., 2000; Peirce et al., 2000; Stice et al., 2004; Symister and Friend, 2003; Paykel et al., 1971) as inversely associated with subsequent major depression. The protective contribution of frequent religious services

\footnotetext{
* Correspondence to: Department of Psychiatry, Columbia University, New York State Psychiatric Institute, Unit 24, NY 10032, USA. Tel.: +1 212543 5880; fax: +12125683534.

E-mail address: weissman@nyspi.columbia.edu (M.M. Weissman).
}

attendance has in part been attributed to the social support that may be present (Hayward et al., 2012b; Koenig et al., 1992; Strawbridge et al., 1997), but its association with depression independent from various social factors has received little investigation. Indeed, it is possible that attending religious services may be closely linked with positive social contact with acquaintances, friends, coworkers, and immediate and extended family members. To date, there is yet to be a longitudinal study to examine the protective effects of frequent religious services attendance against depression, while accounting for the influence of adjustment within these social domains.

Adult offspring of depressed as compared with nondepressed parents are at elevated lifetime risk for major depression (MDD), and show differential risk and protective factors against MDD as compared with adult offspring of nondepressed parents 
(Weissman et al., 2006). Using data from a longitudinal multigeneration study, we had the opportunity to examine the independent effects of frequent religious services attendance and social adjustment on depression by familial risk for depression, in a series of exploratory analyses.

We predict (1) that a high frequency of religious services attendance will protect against subsequent depression, independent of the impact of high social adjustment, and (2) that a family history of depression will attenuate the protective effects of frequent religious services attendance against depression due to an elevated risk for depression.

\section{Method}

The data for this study comes from the 10- (Time 10) and 20(Time 20) year follow-up phases of a multi-wave longitudinal study of women at high and low risk for depression (G1), their offspring (G2), and grandchildren (G3). A full description of the present study has been published elsewhere (Weissman et al., 2006).

The current study focused upon the offspring (G2), because the original probands (G1) were not interviewed at 20-year followup, and the grandchildren (G3) were too young at 10-year followup to obtain reliable information on independent religious attendance. In the original wave of the study, probands with moderate to severe major depressive disorder were selected from outpatient clinics for the psychopharmacologic treatment of mood disorders and their offspring (G2) represent a group at high risk for depression. Nondepressed, matched probands were selected at the same time from an epidemiological sample of adults from the same community and their offspring (G2) represent a group at low risk for depression.

\subsection{Study participants}

At initial assessment 220 offspring of the original probands (G2) between the ages of 6 and 23 years from 91 families were interviewed. The current study concerns 173 offspring who at 10- and 20-year follow-up were present, willing to be interviewed, and had information on their social adjustment, religious attendance, and depression episode.

\subsection{Assessments}

Diagnosis of MDD at 10- and 20-year follow-ups were assessed using the Schedule for Affective Disorders and SchizophreniaLifetime Version for adults (Mannuzza et al., 1986). An indication of MDD at 10-year follow-up represents an episode of MDD between Times 1 and 10, and an indication of MDD at 20-year follow-up represents an episode of MDD between Times 10 and 20.

Frequency of religious services attendance was assessed by self-report at 10-year follow-up. Attendance was categorized as frequent if it occurred at least once a month, based on the review by Larson and Larson (1994) showing a protective effect against psychopathology with at least monthly attendance at religious services.

Social functioning was measured at both 10-year and 20-year follow-up through self-report on the Social Adjustment ScaleSelf Report (SAS-SR) (Gameroff et al., 2012). The SAS-SR measures social adjustment in the areas of the work role, social and leisure, extended family, primary relationship, parental, and family unit. An overall score is calculated, as well as subscores for each of the six areas of social adjustment, with lower scores indicating less impaired social adjustment. Social adjustment was categorized as high functioning for overall scores and subscores on the SAS-SR that fell below that score's mean for the total sample.

\subsection{Statistical analysis}

Univariate and multivariate logistic regression analyses were used to assess the relationship between the frequency of religious services attendance and SAS-SR variables at Time 10 and MDD between Times 10 and 20 (controlling for age, sex, risk group and depression status prior to Time 10). Analyses were then repeated with the data stratified by high and low risk for depression based upon parental depression status.

\section{Results}

\subsection{Sample characteristics}

Table 1 shows the demographic and clinical variables of the 173 participants, by high and low risk group and the overall sample. $\chi^{2}$ tests conducted on differences in demographic characteristics and study variables between risk groups showed differences in Time 10 rates of major depression, frequency of religious services attendance, and social adjustment. The rate of major depression prior to Time 10 was four times greater in the high-risk group than the low-risk group (28.2\% [34/118] compared with $7.3 \%$ [4/55]; $\left.\chi^{2}=10.16, \mathrm{df}=1, p<0.01\right)$. The rate of frequent religious services attendance at Time 10 was significantly greater in the low-risk group than the high-risk group (52.7\% [29/55] compared with $28.0 \%$ [33/118]; $\chi^{2}=10.00, \mathrm{df}=1, p<0.01$ ). Mean overall scores on the SAS-SR were higher, and thus indicated significantly greater impairment, among the high risk group than in the low risk group $(M=1.77, \mathrm{SD}=0.40$ compared with $M=1.61$, $\mathrm{SD}=0.32 ; t(171)=2.57, p<0.05)$; when examined dichotomously (cut-off of 1.72 derived by the overall mean), the rate of high functioning social adjustment at Time 10 was significantly greater in the low-risk group than the high-risk group $(67.3 \%$ [37/55] compared with 50.0\% [59/118]; $\left.\chi^{2}=4.53, \mathrm{df}=1, p<0.05\right)$. Mean SAS-SR subscores were also significantly more impaired in the high risk group than the low risk group in the areas of work role $(M=1.74, \quad \mathrm{SD}=0.95 \quad$ compared with $M=1.45, \quad \mathrm{SD}=0.60$; $t(165)=2.11, p<0.05)$, extended family $(M=1.65, \mathrm{SD}=0.46 \mathrm{com}-$ pared with $M=1.46, \mathrm{SD}=0.37 ; t(171)=2.58, p<0.05)$, and primary relationship $(M=1.86, \mathrm{SD}=0.56$ compared with $M=1.59$, $\mathrm{SD}=0.35 ; t(100)=2.49, p<0.05)$.

The top section of Table 2 lists, for the Time 10 SAS-SR and frequency of religious services attendance variables, the odds ratio for major depression at 20-year follow-up while controlling for age, sex, risk status, and prior depression of offspring in the full sample. Participants who attended religious services at least once a month, compared with other participants, had a lower risk of having an episode of major depression between Times 10 and 20 on a trend level (odds ratio $=0.399, C I=0.157-1.011$ ). Participants with high functioning SAS-SR overall scores (scores falling below the mean), compared with other participants, had a significantly lower risk of having an episode of major depression between Times 10 and 20 (odds ratio $=0.249, \mathrm{CI}=0.105-0.591$ ). When the SAS-SR was further examined by an individual subscore, high functioning social adjustment within the extended family (odds ratio $=0.364, \mathrm{CI}=0.156-0.852, p<0.05$ ), primary relationship (odds ratio $=0.306, \mathrm{CI}=0.106-0.883, p<0.05$ ), and family unit (odds ratio $=0.188, \mathrm{CI}=0.080-0.441, p<0.001$ ) was associated with lower risk for major depression. Findings from the univariate and multivariate logistic regressions were of comparable magnitude and significance. 
Table 1

Demographics, religiosity variables, and clinical characteristics of sample $(N=173)$.

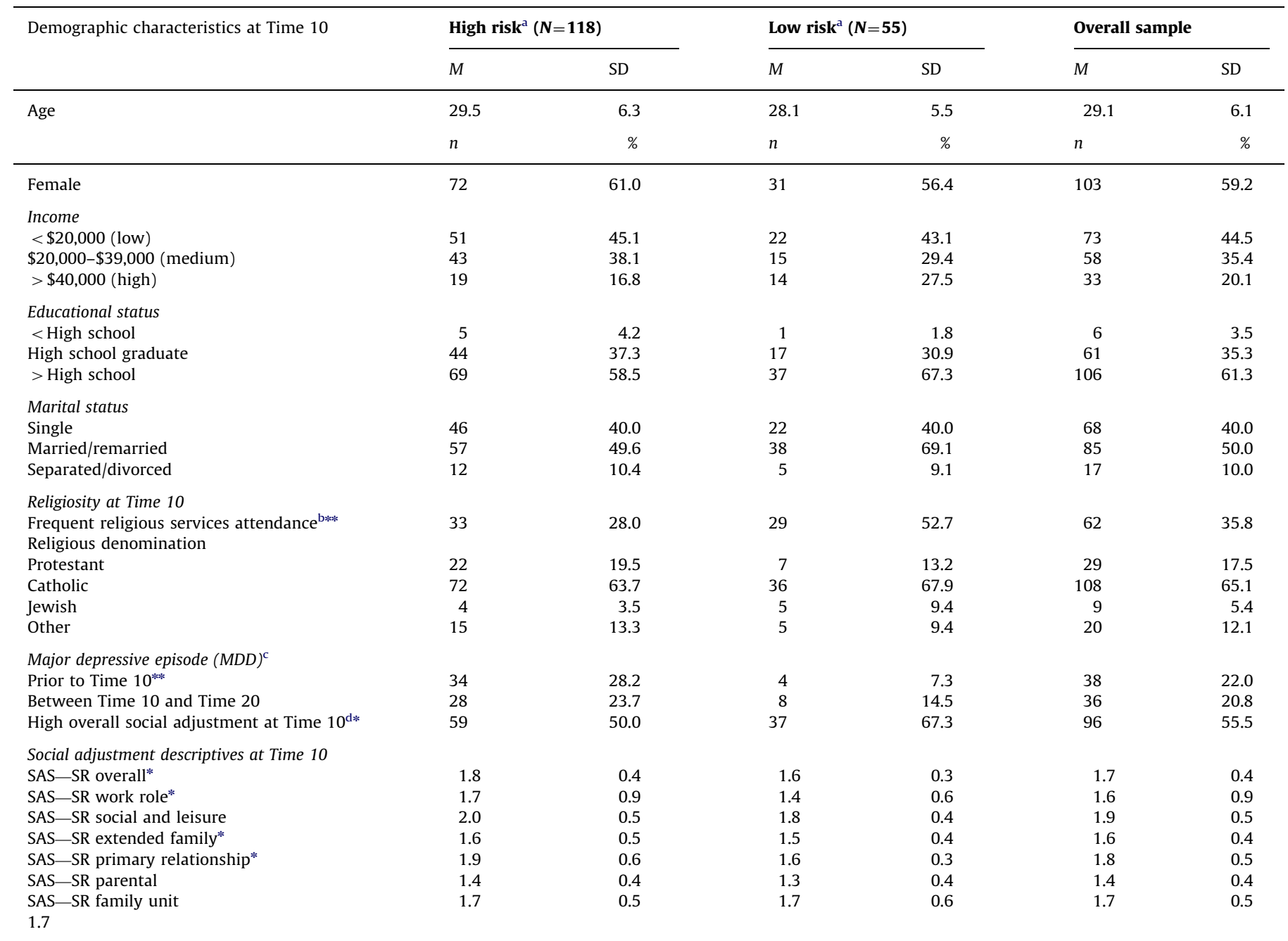

Note: $N$ 's and \% vary due to missing data. ${ }^{a}$ High and low risk group distributions compared with $\chi^{2}$ test and means compared with independent samples $t$-test.

*** $p<0.01$.

$* p<0.05$.

${ }^{a}$ High vs. low risk based on whether either parent did vs. did not have lifetime major depressive disorder.

${ }^{b}$ Attends church at least once a month.

c Assessed with the Schedule for Affective Disorders and Schizophrenia-Lifetime Version.

${ }^{\mathrm{d}}$ Assessed with the Social Adjustment Scale-Self Report High social adjustment determined by scoring above the mean (1.72) of the total sample.

The lower part of Table 2 provides the results of repeating these analyses separately in the high and low risk groups. In the high-risk group, no significant association was found between frequency of religious services attendance and major depression between Times 10 and 20. In the high-risk group, participants with high functioning SAS-SR scores (scores falling below the mean), compared with other participants, had a significantly lower risk of having an episode of major depression between Times 10 and 20 (odds ratio $=0.193, \mathrm{CI}=0.067-0.557$ ). In the lowrisk group, participants who attended religious services at least once a month, compared with other participants, had a lower risk of having an episode of major depression between Times 10 and 20 on a trend level (odds ratio $=0.169, \mathrm{CI}=0.024-1.185$ ). In the low risk group, no significant association was found between SAS-SR and major depression between Times 10 and 20. Findings from the univariate and multivariate logistic regressions were of comparable magnitude and significance.

Within the high risk group, high functioning social adjustment within the extended family (odds ratio $=0.211, \mathrm{CI}=0.072-0.616$, $p<0.01$ ), and family unit (odds ratio $=0.114, \mathrm{CI}=0.053-0.392$, $p<0.001$ ) was associated with lower risk for major depression. No subscales were found to be significantly associated with depression in the low risk group. Findings from the univariate and multivariate logistic regressions were of comparable magnitude and significance.

\section{Discussion}

The findings of this 10-year longitudinal study suggest that there may be long-term and independent protective effects of both frequent religious services attendance and high functioning social adjustment against major depression. When examined by familial risk for depression, exploratory analyses suggested that frequent attendance was protective against depression in adults with no parental history of depression, while high functioning social adjustment was protective against depression in adults with a depressed parent. 
Table 2

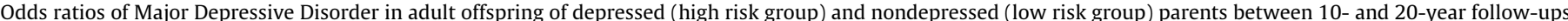

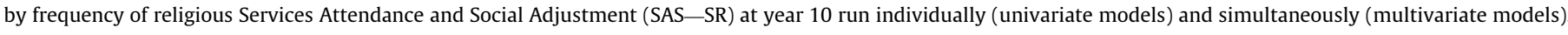

\begin{tabular}{|c|c|c|c|c|c|c|c|c|}
\hline \multirow[t]{2}{*}{ Total sample and risk group } & \multicolumn{4}{|c|}{ Univariate models ${ }^{a}$} & \multicolumn{4}{|c|}{ Multivariate models ${ }^{\mathrm{b}}$} \\
\hline & Odds ratio & $95 \% \mathrm{CI}$ & Wald $\chi^{2}$ & $p$ & Odds ratio & $95 \% \mathrm{CI}$ & Wald $\chi^{2}$ & $p$ \\
\hline \multicolumn{9}{|l|}{ Total sample $(N=173)$} \\
\hline Frequent attendance at religious/spiritual services & 0.454 & $0.184-1.118$ & 2.950 & 0.086 & 0.399 & $0.157-1.011$ & 3.749 & 0.053 \\
\hline \multicolumn{9}{|l|}{ High social adjustment (SAS-SR) } \\
\hline Overall & 0.268 & $0.114-0.629$ & 9.158 & 0.002 & 0.249 & $0.105-0.591$ & 9.928 & 0.002 \\
\hline Work role & 0.607 & $0.259-1.423$ & 1.320 & 0.251 & 0.653 & $0.275-1.546$ & 0.940 & 0.332 \\
\hline Social and leisure & 0.467 & $0.214-1.021$ & 3.639 & 0.056 & 0.450 & $0.203-0.996$ & 3.884 & 0.049 \\
\hline Extended family & 0.364 & $0.156-0.852$ & 5.426 & 0.020 & 0.348 & $0.148-0.823$ & 5.787 & 0.016 \\
\hline Primary relationship & 0.306 & $0.106-0.883$ & 4.792 & 0.029 & 0.287 & $0.097-0.848$ & 5.103 & 0.024 \\
\hline Parental & 0.340 & $0.078-1.483$ & 2.060 & 0.151 & 0.445 & $0.089-2.230$ & 0.970 & 0.325 \\
\hline Family unit & 0.188 & $0.080-0.441$ & 15.818 & 0.000 & 0.192 & $0.081-0.453$ & 14.178 & 0.000 \\
\hline \multicolumn{9}{|l|}{ High risk group $(N=118)$} \\
\hline Frequent attendance at religious/spiritual services & 0.553 & $0.190-1.606$ & 1.186 & 0.276 & 0.435 & $0.141-1.345$ & 2.087 & 0.149 \\
\hline High social adjustment overall & 0.215 & $0.076-0.604$ & 8.486 & 0.004 & 0.193 & $0.067-0.557$ & 9.271 & 0.002 \\
\hline \multicolumn{9}{|l|}{ Low risk group $(N=55)$} \\
\hline Frequent attendance at religious/spiritual services & 0.180 & $0.026-1.240$ & 3.032 & 0.082 & 0.169 & $0.024-1.185$ & 3.202 & 0.074 \\
\hline High social adjustment overall & 0.278 & $0.046-1.671$ & 1.958 & 0.162 & 0.247 & $0.037-1.630$ & 2.110 & 0.146 \\
\hline
\end{tabular}

${ }^{\text {a }}$ For each of the 12 univariate models, the outcome measure is major depressive disorder at year 20 (yes/no) and the primary predictors are the frequency of religious

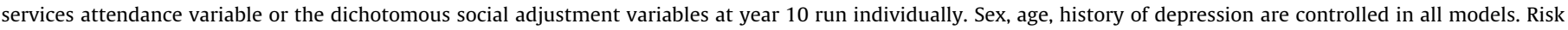
group is controlled for in the total sample analyses.

${ }^{\mathrm{b}}$ For each of the nine multivariate models, the outcome measure is major depressive disorder at year 20 (yes/no) and the primary predictors are the frequency of

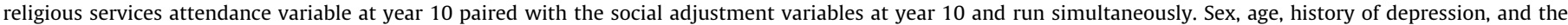
other dichotomous variable are controlled in both models. Risk group is controlled for in the total sample analyses.

Frequency of religious services attendance was negatively associated with major depression at a trend level: adults that attended religious services at least once a month were less likely to become depressed over the 10-year follow up period. This finding persisted when controlling for the effects of social adjustment, suggesting that frequent attendance is protective beyond the various forms of social contact that may be present at religious services.

Previously the protective effects of frequent attendance at religious services have been attributed in part to the presence of positive social factors in religious settings (Hayward et al., 2012b; Koenig et al., 1992; Strawbridge et al., 1997); however these studies have focused primarily on the elderly and medically ill. The present study has found that frequent religious services attendance may be protective against depression in healthy middle-aged adults, regardless of social adjustment among acquaintances, friends, coworkers, and immediate and extended family members. This supports previous findings that frequent religious attendance protects against depression in middle-aged women, independent from the social interaction present at religious services (Hintikka et al., 1998).

When examined by familial risk for depression, the protective benefits of frequent attendance against MDD were found primarily in adults with low familial risk for depression. This suggests that the consistent finding in existing literature on the protective qualities of frequent attendance (Koenig, 2007; Maselko et al., 2009; Norton et al., 2008; Sternthal et al., 2010) may pertain more often to adults at low familial risk for depression, in light of the current finding.

High functioning social adjustment was negatively associated with major depression over 10 years. When investigated by specific areas of social adjustment, this effect was located particularly within the extended family, primary relationship, and family unit. Among adult offspring of depressed parents, those that had high functioning social adjustment were significantly less likely to have an episode of depression over the 10-year follow-up period, compared with others in the high-risk group. This effect was located primarily within extended family and family unit. These findings were stable when controlling for frequency of religious services attendance. No protective benefit was found of high functioning social adjustment against depression among adults at low familial risk for depression. This may be explained by the lower average level of impairment in social adjustment among the low risk group. Dyer and Giles (1994) found that "within the context of the parent-offspring interaction, increased vulnerability for depression in offspring of depressed parents may be mediated in part by the enduring impact of social adjustment" . This supports the present finding that high social adjustment within the family is associated with a lower risk for major depression, especially among those with depressed parents.

The present study contained several limitations: (1) Our use of the SAS-SR to measure social adjustment was not limited to religious services attendance, instead measuring adjustment in a broad range of social domains. (2) Our measure of frequency of religious services attendance relied on self-report, thus depending on participants to accurately report their attendance. (3) The longitudinal high-risk design of the present study required recruitment of offspring of depressed and nondepressed parents and assessment over 10 years, thus the sample size is smaller than can be obtained through other study designs.

Overall this study found that over 10 years, frequent attendance at religious services may protect against depression independent of social adjustment. Exploratory analyses suggest that these findings may be limited to adults with low familial risk for depression. Adults at high familial risk for depression may derive protective benefit from overall social functioning and within the family, but not religious attendance. Future research might further examine the specific protective components of religious services attendance and familial social adjustment within the context of parental depression. Clinical practice and pastoral counseling might integrate awareness of family depression history when developing supports.

\section{Role of funding source}

The funding sources had no involvement in the study design, in collection, analysis and interpretation of data, in writing the report, and in the decision to submit the paper for publication. 


\section{Conflict of interest}

All other authors declare that they have no conflicts of interest.

\section{Financial disclosure}

In the past 2 years Dr. Miller received funding from the John Templeton Foundation, the Klingenstein Fund and the Pritchard Foundation; and received payment from Oxford University Press. Dr. Weissman received funding from the National Institute of Mental Health (NIMH), the National Alliance for Research on Schizophrenia and Depression (NARSAD), the Sackler Foundation, the John Templeton Foundation and the Interstitial Cystitis Association; and receives royalties from the Oxford University Press, Perseus Press, and the American Psychiatric Association Press. Dr. Weissman receives royalties from MultiHealth Systems for the Social Adjustment Scale.

\section{Acknowledgment}

The analyses were funded by the John Templeton Foundation. The data collection was funded by the National Institute of Mental Health (2 R01 MH36197).

\section{References}

Bruce, M.L., Hoff, R.A., 1994. Social and physical health risk factors for first-onse major depressive disorder in a community sample. Social Psychiatry and Psychiatric Epidemiology 29, 165-171.

De Leeuw, J.R.J., De Graeff, A., Ros, W.J.G., Hordijk, G.J., Blijham, G.H., Winnubst, J.A.M., 2000. Negative and positive influences of social support on depression in patients with head and neck cancer: a prospective study. Psycho-Oncology 9, 20-28.

Dyer, J.G., Giles, D.E., 1994. Familial influence in unipolar depression: effects of parental cognitions and social adjustment on adult offspring. Comprehensive Psychiatry 35, 290-295.

Gameroff, M.J., Wickramaratne, P., Weissman, M.M., 2012. Testing the short and screener versions of the Social Adjustment Scale-Self-report (SAS-SR) International Journal of Methods in Psychiatric Research 21, 52-65.

Hayward, R.D., Owen, A.D., Koenig, H.G., Steffens, D.C., Payne, M.E., 2012b. Longitudinal relationships of religion with posttreatment depression severity in older psychiatric patients: evidence of direct and indirect effects. Depression Research and Treatment 2012, 1-8.

Hayward, R.D., Owen, A.D., Koenig, H.G., Steffens, D.C., Payne, M.E., 2012a. Religion and the presence and severity of depression in older adults. American Journal of Geriatric Psychiatry 20, 188-192.

Hintikka, J., Viinamaki, H., Koivumaa-Honkanen, H., Tanskanen, A., Lehtonen, J. 1998. Associations between religious attendance, social support, and depression in psychiatric patients. Journal of Psychology and Theology 26, 351-357.

Koenig, H.G., 2007. Religion and depression in older medical inpatients. American Journal of Geriatric Psychiatry 15, 282-291.

Koenig, H.G., Cohen, H.J., Blazer, D.G., Pieper, C., Meador, K.G., Shelp, F., Goli, V., DiPasquale, B., 1992. Religious coping and depression among elderly, hospitalized medically ill men. American Journal of Psychiatry 149, 1693-1700.

Larson, D.B., Larson, S.S., 1994. The Forgotten Factor in Physical and Mental Health: What Does the Research Show? Rockville, Md, National Institute for Healthcare Research

Mannuzza, S., Fyer, A.J., Klein, D.F., Endicott, J., 1986. Schedule for affective disorders and schizophrenia-lifetime version modified for the study of anxiety disorders (SADS-LA): rationale and conceptual development. Journal of Psychiatric Research 20, 317-325.

Maselko, J., Gilman, S.E., Buka, S., 2009. Religious service attendance and spiritual well-being are differentially associated with risk of major depression. Psychological Medicine 39, 1009-1017.

Norton, M.C., Singh, A., Skoog, I., Corcoran, C., Tschanz, J.T., Zandi, P.P., Breitner, J.C.S., Welsh-Bohmer, K.A., Steffens, D.C., 2008. Church attendance and new episodes of major depression in a community study of older adults: the cache county study. Journals of Gerontology 63B, 129-137.

Paykel, E.S., Weissman, M., Prusoff, B.A., Tonks, C.M., 1971. Dimensions of social adjustment in depressed women. Journal of Nervous and Mental Disease 152, $158-172$.

Peirce, R.S., Frone, M.R., Russell, M., Cooper, M.L., Mudar, P., 2000. A longitudinal model of social contact, social support, depression, and alcohol use. Health Psychology 19, 28-38.

Sternthal, M.J., Williams, D.R., Musick, M.A., Buck, A.C., 2010. Depression, anxiety, and religious life: a search for mediators. Journal of Health and Social Behavior 51, 343-359.

Stice, E., Ragan, J., Randall, P., 2004. Prospective relations between social support and depression: differential direction of effects for parent and peer support? Journal of Abnormal Psychology 113, 155-159.

Strawbridge, W.J., Cohen, R.D., Shema, S.J., Kaplan, G.A., 1997. Frequent attendance at religious services and mortality over 28 years. American Journal of Public Health 87, 957-961.

Symister, P., Friend, R., 2003. The influence of social support and problematic support on optimism and depression in chronic illness: a prospective study evaluating self-esteem as a mediator. Health Psychology 22, 123-129.

Weissman, M.M., Wickramaratne, P., Nomura, Y., Warner, V., Pilowsky, D., Verdeli, H., 2006. Offspring of depressed parents: 20 years later. American Journal of Psychiatry 163, 1001-1008. 